(2) OPEN ACCESS

- Additional supplemental material is published online only. To view, please visit the journal online (http://dx doi.org/10.1136/jnnp-2021327412).

For numbered affiliations see end of article.

Correspondence to Professor Friedemann Paul Experimental and Clinical Research Center, Charité Universitätsmedizin Berlin, 10117 Berlin, Germany; friedemann.paul@charite.de

$A B$ and $F C O$ contributed equally.

Received 24 June 2021 Accepted 26 September 2021 Published Online First 28 October 2021

\section{Linked}

- http://dx.doi.org/10.1136/ jnnp-2021-327846

Check for updates

(C) Author(s) (or their employer(s)) 2022. Re-use permitted under CC BY-NC. No commercial re-use. See rights and permissions. Published by BMJ.

To cite: Lu A, Zimmermann HG, Specovius S, et al. J Neurol Neurosurg Psychiatry 2022:93:188-195.

\title{
Astrocytic outer retinal layer thinning is not a feature in AQP4-IgG seropositive neuromyelitis optica spectrum disorders
}

\author{
Angelo Lu (D) ,1,2 Hanna G Zimmermann, 1,2 Svenja Specovius, 1,2
} Seyedamirhosein Motamedi, ${ }^{1,2}$ Claudia Chien (10 , 1,2 Charlotte Bereuter, ${ }^{1,2}$ Marco A Lana-Peixoto, ${ }^{3}$ Mariana Andrade Fontenelle, ${ }^{3}$ Fereshteh Ashtari, ${ }^{4}$ Rahele Kafieh, ${ }^{5}$ Alireza Dehghani, ${ }^{6}$ Mohsen Pourazizi, ${ }^{6}$ Lekha Pandit, Anitha D'Cunha, ${ }^{7}$ Ho Jin Kim (10 ${ }^{8}$ Jae-Won Hyun, ${ }^{8}$ Su-Kyung Jung ${ }_{1}^{9}$ Letizia Leocani, ${ }^{10}$ Marco Pisa, ${ }^{10}$ Marta Radaelli, ${ }^{10}$ Sasitorn Siritho, ${ }^{11}$ Eugene F May, ${ }^{12}$ Caryl Tongco, ${ }^{12}$ Jérôme De Sèze, ${ }^{13}$ Thomas Senger, $^{13}$ Jacqueline Palace, ${ }^{14}$

Adriana Roca-Fernández (D) , ${ }^{14}$ Maria Isabel Leite, ${ }^{14}$ Srilakshmi M Sharma, ${ }^{15}$ Hadas Stiebel-Kalish (1) ${ }^{16,17}$ Nasrin Asgari, ${ }^{18}$ Kerstin Kathrine Soelberg, ${ }^{19}$ Elena H Martinez-Lapiscina (D) ${ }^{20}$ Joachim Havla (D) , ${ }^{21}$ Yang Mao-Draayer, ${ }^{22}$ Zoe Rimler, ${ }^{23}$ Allyson Reid, ${ }^{23}$ Romain Marignier, ${ }^{24}$ Alvaro Cobo-Calvo, ${ }^{24,25}$ Ayse Altintas, ${ }^{26}$ Uygur Tanriverdi, ${ }^{27}$ Rengin Yildirim, ${ }^{28}$ Orhan Aktas, $^{29}$ Marius Ringelstein (D) , ${ }^{29,30}$ Philipp Albrecht (1) , ${ }^{29}$ Ivan Maynart Tavares, ${ }^{31}$ Denis Bernardi Bichuetti (D) , ${ }^{32}$ Anu Jacob, ${ }^{33}$ Saif Huda ${ }^{33}$ Ibis Soto de Castillo, ${ }^{34}$ Axel Petzold (D) , ${ }^{35}$ Ari J Green, ${ }^{36}$ Michael R Yeaman, ${ }^{37,38}$ Terry J Smith, ${ }^{39,40}$ Lawrence Cook, ${ }^{41}$ Friedemann Paul, ${ }^{1,2,42}$ Alexander U Brandt, 1,2,43 Frederike Cosima Oertel (D) , ${ }^{1,2,36}$ GJCF International Clinical Consortium for NMOSD

\section{ABSTRACT}

Background Patients with anti-aquaporin-4 antibody seropositive (AQP4-lgG+) neuromyelitis optica spectrum disorders (NMOSDs) frequently suffer from optic neuritis (ON) leading to severe retinal neuroaxonal damage. Further, the relationship of this retinal damage to a primary astrocytopathy in NMOSD is uncertain. Primary astrocytopathy has been suggested to cause $\mathrm{ON}$ independent retinal damage and contribute to changes particularly in the outer plexiform layer (OPL) and outer nuclear layer (ONL), as reported in some earlier studies. However, these were limited in their sample size and contradictory as to the localisation. This study assesses outer retinal layer changes using optical coherence tomography $(\mathrm{OCT})$ in a multicentre cross-sectional cohort.

Method 197 patients who were AQP4-lgG+ and 32 myelin-oligodendrocyte-glycoprotein antibody seropositive (MOG-IgG+) patients were enrolled in this study along with 75 healthy controls. Participants underwent neurological examination and OCT with central postprocessing conducted at a single site.

Results No significant thinning of OPL $(25.02 \pm 2.03$ $\mu \mathrm{m})$ or ONL $(61.63 \pm 7.04 \mu \mathrm{m})$ were observed in patients who were AQP4-IgG + compared with patients who were MOG-lgG + with comparable neuroaxonal damage (OPL: $25.10 \pm 2.00 \mu \mathrm{m}$; ONL: $64.71 \pm 7.87 \mu \mathrm{m}$ ) or healthy controls (OPL: $24.58 \pm 1.64 \mu \mathrm{m} ;$ ONL: $63.59 \pm 5.78 \mu \mathrm{m})$. Eyes of patients who were AQP4-lgG+ $(19.84 \pm 5.09 \mu \mathrm{m}$, $\mathrm{p}=0.027)$ and MOG- $\lg G+(19.82 \pm 4.78 \mu \mathrm{m}, \mathrm{p}=0.004)$ with a history of ON showed parafoveal OPL thinning compared with healthy controls $(20.99 \pm 5.14 \mu \mathrm{m})$; this was not observed elsewhere.

Conclusion The results suggest that outer retinal layer loss is not a consistent component of retinal astrocytic damage in AQP4-lgG+ NMOSD. Longitudinal studies are necessary to determine if OPL and ONL are damaged in late disease due to retrograde trans-synaptic axonal degeneration and whether outer retinal dysfunction occurs despite any measurable structural correlates.

\section{INTRODUCTION}

Neuromyelitis optica spectrum disorders (NMOSDs) are relapsing autoimmune disorders affecting the central nervous system (CNS). ${ }^{1}$ Common clinical attacks in NMOSD include optic neuritis $(\mathrm{ON})$, acute myelitis and area postrema syndrome. ${ }^{2}$ Serum autoantibodies to aquaporin-4 (AQP4-IgG) are detectable in $60 \%-80 \%$ of patients with NMOSD . ${ }^{34}$

AQP4 is an astrocytic water channel in the CNS. ${ }^{5}$ In the retina, astrocytes are mainly located in the inner neuroaxonal layers of the retina, but AQP4 is additionally highly expressed in retinal Müller cells. ${ }^{6}$ These glial cells have diverse functions, such as regulation of water homeostasis and neurotransmitter recycling, and are located around the fovea 


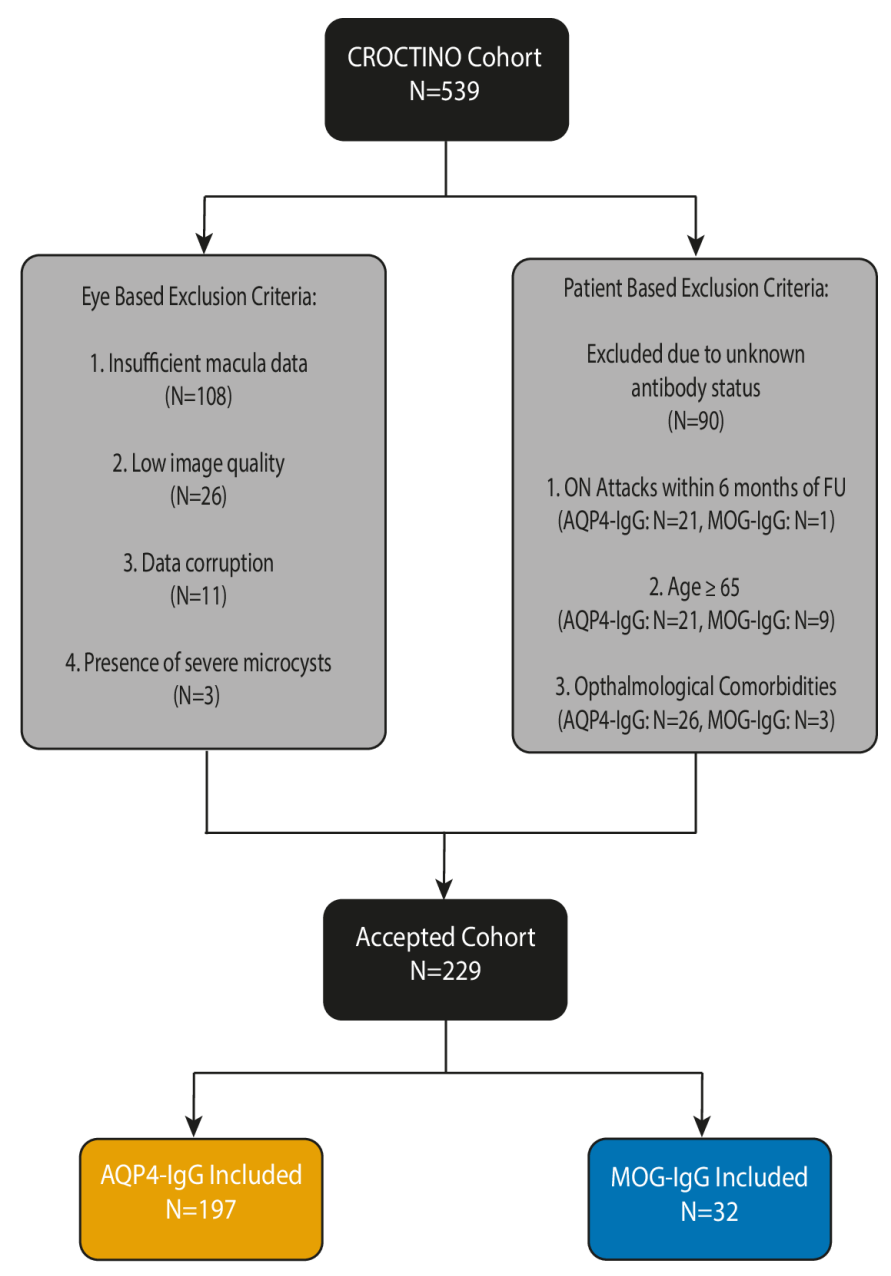

HC Included $\mathrm{N}=75$

Figure 1 Cohort design and exclusion criteria: from the original 539 patients recruited in the CROCTINO cohort, 108 patients were excluded due to missing macular data. Of the remaining 431 patients in the segmentation cohort, a further 40 patients were excluded due to anomalies in their OCT scans (OSCAR-IB criteria; primarily due to low image quality (26 patients) or the presence of microcysts (3 patients) or other pathologies) or due to data corruption (11 patients). We also excluded patients with unknown antibody status (90 patients). Of the remaining 301 patients, the cohort was split based on AQP4-IgG or MOG-IgG seropositivity and a further set of exclusion criteria were applied based on age (being $\geq 65$ years), ophthalmological comorbidities (eg, glaucoma) and in instances where follow-ups occurred within 6 months of an ON attack. AQP4-IgG, anti-aquaporin-4 antibody; HC, healthy control; MOG-IgG, anti-myelin-oligodendrocyte-glycoprotein antibody; OCT, optical coherence tomography; ON, optic neuritis.

spanning the entire thickness of the retina. ${ }^{7}$ Of particular interest is also the Henle Fibre outer nuclear layer (ONL) boundary of the parafovea where AQP4 channels are highly expressed. ${ }^{8}$

A primary and attack-independent astrocytopathy in NMOSD has been suggested to contribute to retinal neurodegeneration and to Müller cell-associated parafoveal changes. ${ }^{9-13}$ Recent studies suggested potential astrocytopathy-related outer retinal layer (ORL) thinning in AQP4-IgG seropositive NMOSD but were limited in their sample size and in parts contradictory on the exact layers in which these changes occur. ${ }^{811}$ It thereby remains unclear if ORLs, especially the ONL are also potentially affected by primary retinal astrocytopathy in AQP4-IgG seropositive NMOSD.

Representing the largest international NMOSD dataset collected so far, the CROCTINO study (Collaborative Retrospective Study on retinal optical coherence tomography (OCT) in Neuromyelitis Optica) overcomes one of the common weaknesses of NMOSD studies-being limited to small and homogenous sample populations. ${ }^{14} 15$ Using OCT data from over 20 centres worldwide, reliable quantitative and qualitative retinal assessment becomes possible, and controversial questions such as ORL changes in AQP4-IgG seropositive NMOSD can be clarified. Apart from patients who were AQP4-IgG seropositive, the CROCTINO cohort also includes patients with antibodies to myelin-oligodendrocyte-glycoprotein (MOG-IgG); a group that is now believed to be a distinct disease entity. ${ }^{14-18}$ While clinically similar and undergoing comparable retinal neurodegeneration after ON, MOG-IgG-associated disease (MOGAD) lacks an identifiable astrocytopathy component and is thereby an appropriate diseased control group for patients who were AQP4-IgG seropositive when investigating astrocytic changes. ${ }^{10} 19$

In this study, we investigated if ORL thinning, specifically in the foveal and macular ONL, occurs in patients who were AQP4-IgG seropositive compared with healthy controls (HCs) and with patients with MOGAD as a diseased control group.

\section{METHODS}

\section{Cohort design}

A total of 539 patients with NMOSD were recruited between 2000 and 2018 as part of CROCTINO (stratified data of centres by device type and number of patients are summarised in the online supplemental file 1). ${ }^{14}$ Patients with (1) diseases potentially confounding OCT analyses (including glaucoma, diabetic retinopathy, retinal surgery and ametropia greater than \pm 6 diopters), (2) a history of ON within the last 6 months before baseline, (3) no evidence of seropositivity for AQP4-IgG or MOG-IgG 2021 and (4) no macular OCT data were excluded. Cell-based assays were used for the detection of AQP4-IgG and MOG-IgG antibodies in serum samples from all patients. Clinical data (antibody serology, disease duration, frequency of $\mathrm{ON}$, location of ON, date of ON, Expanded Disability Standard Scale and treatment received) were collected from all patients. We also included 75 HCs (recruited from Barcelona, Isfahan, Mangalore and Berlin), who were neither age nor sex matched to either cohort.

\section{Optical coherence Tomography}

Retinal examinations were conducted at each centre using the following OCT devices: Spectralis SD-OCT, Heidelberg Engineering, Heidelberg, Germany (Spectralis), Cirrus HD-OCT, Carl Zeiss Meditec Inc, Dublin, California, USA (Cirrus) and Topcon 3D-OCT, Topcon Corp, Tokyo, Japan (Topcon). With respect to each device and each centre, two scans were collected: (1) a $3.4 \mathrm{~mm}$ diameter peripapillary ring scan around the optic nerve head for Spectralis SD-OCT (for Cirrus and Topcon devices: extracted from optic disc volume scans), and (2) a macular volume scans, centred on the fovea. ${ }^{14}$ Scans were categorised and uploaded onto a central server to be accessed for further processing.

All OCT images fulfilled the OSCAR-IB criteria ${ }^{22} 23$ (see figure 1-images from 29 patients not fulfilling these criteria were excluded) and results were presented in line with the 
Table 1 Demographic overview

\begin{tabular}{|c|c|c|c|}
\hline & $\mathrm{HC}$ & AQP4-IgG & MOG-IgG \\
\hline $\begin{array}{l}\text { Subjects } \\
\text { (N) }\end{array}$ & 75 & 197 & 32 \\
\hline $\begin{array}{l}\text { Number of eyes } \\
\text { (N) }\end{array}$ & 148 & 317 & 55 \\
\hline $\begin{array}{l}\text { Age } \\
\text { (years, mean } \pm S D \text { ) }\end{array}$ & $32.3 \pm 9.6$ & $41.8 \pm 12.1$ & $36.5 \pm 13.7$ \\
\hline $\begin{array}{l}\text { Sex } \\
(\text { male, N (\%)) }\end{array}$ & $25(33.8)$ & $24(12.2)$ & $10(31.2)$ \\
\hline $\begin{array}{l}\text { EDSS } \\
\text { (median (IQR)) }\end{array}$ & - & $3.5(2.0-5.0)$ & $2.0(1.5-2.5)$ \\
\hline $\begin{array}{l}\text { Average age at onset } \\
\text { (years, median (IQR)) }\end{array}$ & - & $32.9(24.9-42.4)$ & $30.0(17.6-42.5)$ \\
\hline $\begin{array}{l}\text { Patients with a history } \\
\text { of ON } \\
(\mathrm{N}(\%))\end{array}$ & - & $142(72.1)$ & $24(75.0)$ \\
\hline $\begin{array}{l}\text { Median number of ON } \\
\text { episodes (median, IQR) }\end{array}$ & - & $1.00(0.00-3.00)$ & $2.00(1.00-4.00)$ \\
\hline $\begin{array}{l}\text { Disease duration } \\
\text { (years, mean } \pm S D \text { ) }\end{array}$ & - & $7.1 \pm 6.7$ & $4.8 \pm 7.8$ \\
\hline $\begin{array}{l}\text { Ethnicity } \\
(\mathrm{N}(\%))\end{array}$ & $\begin{array}{l}\text { White }(57(761)) \\
\text { Asian }(16(21.3)) \\
\text { Hispanic }(1(1.3)) \\
\text { Other (1 (1.3)) }\end{array}$ & $\begin{array}{l}\text { White (105 (53.3) } \\
\text { Asian (56 (28.4)) } \\
\text { African American (11 (5.6)) } \\
\text { Other (25 (12.7)) }\end{array}$ & $\begin{array}{l}\text { White }(19(59.4)) \\
\text { Asian }(13(40.6))\end{array}$ \\
\hline $\begin{array}{l}\text { Current treatment } \\
(\mathrm{N}(\%))\end{array}$ & & $\begin{array}{l}\text { Rituximab }(51(25.9)) \\
\text { Azathioprine }(42(21.3)) \\
\text { Mycophenolate Mofetil } \\
\text { (31 (15.7)) } \\
\text { Methotrexate }(4(2.0)) \\
\text { Other or missing }(69(35.0))\end{array}$ & $\begin{array}{l}\text { Rituximab (6 (18.8)) } \\
\text { Azathioprine (6 (18.8)) } \\
\text { Prednisone (6 (18.8)) } \\
\text { Mycophenolate } \\
\text { mofetil (5 (15.6)) } \\
\text { Other or missing (9 } \\
(28.1))\end{array}$ \\
\hline OCT device (N (\%)) & $\begin{array}{l}\text { Spectralis (75 } \\
(100))\end{array}$ & $\begin{array}{l}\text { Spectralis }(139(70.6)) \\
\text { Cirrus }(38(19.3)) \\
\text { Topcon }(20(10.2))\end{array}$ & $\begin{array}{l}\text { Spectralis }(25(78.1)) \\
\text { Cirrus }(3(9.4)) \\
\text { Topcon }(4(12.5))\end{array}$ \\
\hline \multicolumn{4}{|c|}{$\begin{array}{l}\text { Cirrus: Cirrus HD-OCT, Carl Zeiss Meditec Inc, Dublin, California, USA; Spectralis: SD-OCT, Heidelberg } \\
\text { Engineering, Heidelberg, Germany;Topcon: Topcon 3D-OCT, Topcon Corp, Tokyo Japan. } \\
\text { AQP4-IgG, anti-aquaporin-4 antibody; EDSS, Expanded Disability Standard Scale; HCs, healthy controls; } \\
\text { MOG-IgG, anti-myelin-oligodendrocyte-glycoprotein antibody; N, number of subjects; ON, optic neuritis. }\end{array}$} \\
\hline
\end{tabular}

APOSTEL V.2.0 recommendations. ${ }^{24}$ Peripapillary retinal nerve fibre layer ( $\mathrm{pRNFL}$ ) thickness was derived using a device-specific protocol and centred around the optic nerve head. Segmentation of all layers in macular volume scans were performed semiautomatically and processed with an in-house proprietary software
(SAMIRIX). ${ }^{25}$ For the purposes of this study, the macular retinal layers were segmented in the following layers: macular retinal nerve fibre layer (mRNFL), ganglion cell and inner plexiform layer (GCIP), inner nuclear layer (INL), outer plexiform layer (OPL), ONL, the outer plexiform and nuclear layer (OPNL), photoreceptor layer (PR, inner photoreceptor segments to Bruch's membrane) and the total retinal thickness (RT, calculated as the thickness consisting of the RNFL (defined as layer no. 3 per Staurenghi et $a l^{26}$ ) to the Bruch's membrane (layer no. 14). All scans were checked and, where necessary, manual correction of the automatic segmentation was conducted using SAMIRIX by experienced raters (FCO, CB and SS for ring scans, HZ, FCO and AL for macular scans) at a single site at the Charité-Universitätsmedizin Berlin. To assure comparability with previously published data on ORL changes in NMOSD, the macular volume data were further segregated into one of three export protocols: (1) a $5 \mathrm{~mm}$ diameter cylinder omitting a $1 \mathrm{~mm}$ diameter around the fovea (5 mm study), (2) a $3 \mathrm{~mm}$ diameter cylinder omitting a $1 \mathrm{~mm}$ diameter around the fovea $(3$ mm study) and (3) a $1 \mathrm{~mm}$ mean thickness around the fovea (1 mm study). Results are reported for the $5 \mathrm{~mm}$ study on Spectralis devices; confirmatory results based on the $3 \mathrm{~mm}$ and $1 \mathrm{~mm}$ study as well as for Cirrus and Topcon devices are set out in the online supplemental file 1 .

\section{Statistical methods}

Data were stratified in cohorts by (1) antibody status and (2) ON history (contralateral eyes of patients with a history of unilateral $\mathrm{ON}$ are classified not fulfilling the $\mathrm{ON}$ history criteria). The data were further bifurcated by OCT device (Spectralis, Cirrus or Topcon) to mitigate any device-specific aberrations. For continuous cohort data (age, average age at onset and disease duration) on each of the AQP4-IgG, MOG-IgG and HC cohorts, the Student's t-test was employed. Cross-sectional group comparisons of the OCT values were conducted using linear mixed-effect models with age and sex as fixed and centre and patient-ID as random effects; where necessary, models were corrected for age and sex. Marginal and conditional coefficients of determination for the models were estimated by pseudo- $\mathrm{R}^{2}$ for mixed-effect

Table 2 Group comparison between HC and patients who were AQP4-IgG and MOG-IgG seropositive at baseline (Spectralis devices only)

\begin{tabular}{|c|c|c|c|c|c|c|c|c|c|c|c|c|}
\hline & \multirow[b]{2}{*}{$\mathrm{HC}$} & \multirow[b]{2}{*}{ AQP4-IgG } & \multirow[b]{2}{*}{ MOG-IgG } & \multicolumn{3}{|c|}{ AQP4-IgG vs HC } & \multicolumn{3}{|c|}{ AQP4-IgG vs MOG-IgG } & \multicolumn{3}{|c|}{ MOG-IgG vs HC } \\
\hline & & & & B & SE & $\mathbf{P}$ & B & SE & $\mathbf{P}$ & B & SE & $\mathbf{P}$ \\
\hline Number of eyes & 148 & 317 & 55 & & & & & & & & & \\
\hline $\begin{array}{l}\text { pRNFL in } \\
\mu \mathrm{m}(\text { mean } \pm \text { SD) }\end{array}$ & $99.17 \pm 9.76$ & $78.46 \pm 24.13$ & $74.33 \pm 23.44$ & -20.22 & 2.86 & $<0.001$ & 0.34 & 4.33 & 0.937 & -29.40 & 2.75 & $<0.001$ \\
\hline $\begin{array}{l}\text { mRNFL in } \\
\mu \mathrm{m}(\text { mean } \pm S D)\end{array}$ & $35.25 \pm 3.13$ & $28.09 \pm 6.60$ & $27.62 \pm 5.43$ & -6.12 & 0.69 & $<0.001$ & -0.15 & 1.38 & 0.913 & -6.98 & 0.66 & $<0.001$ \\
\hline $\begin{array}{l}\text { GCIP in } \\
\mu \mathrm{m} \text { (mean } \pm \text { SD) }\end{array}$ & $80.62 \pm 6.14$ & $65.81 \pm 13.03$ & $66.16 \pm 11.85$ & -14.74 & 1.45 & $<0.001$ & -2.18 & 2.95 & 0.461 & -15.16 & 1.33 & $<0.001$ \\
\hline $\begin{array}{l}\text { INL in } \\
\mu \mathrm{m}(\text { mean } \pm S D)\end{array}$ & $39.64 \pm 2.51$ & $39.85 \pm 3.57$ & $41.55 \pm 4.14$ & 0.34 & 0.39 & 0.384 & -1.93 & 0.87 & 0.028 & 1.79 & 0.53 & 0.001 \\
\hline $\begin{array}{l}\text { OPL in } \\
\mu \mathrm{m}(\text { mean } \pm S D)\end{array}$ & $24.58 \pm 1.64$ & $25.02 \pm 2.03$ & $25.10 \pm 2.00$ & 0.28 & 0.24 & 0.241 & -0.21 & 0.44 & 0.634 & -0.01 & 0.29 & 0.986 \\
\hline $\begin{array}{l}\text { ONL in } \\
\mu \mathrm{m}(\text { mean } \pm \text { SD) }\end{array}$ & $63.59 \pm 5.78$ & $61.63 \pm 7.04$ & $64.71 \pm 7.87$ & -0.01 & 0.83 & 0.993 & -1.77 & 1.80 & 0.327 & 0.69 & 0.93 & 0.457 \\
\hline $\begin{array}{l}\text { OPNL in } \\
\mu \mathrm{m}(\text { mean } \pm S D)\end{array}$ & $89.23 \pm 6.95$ & $86.65 \pm 7.21$ & $89.81 \pm 8.61$ & -0.41 & 0.85 & 0.634 & -1.54 & 1.85 & 0.406 & -0.14 & 0.93 & 0.878 \\
\hline $\begin{array}{l}\text { PR in } \\
\mu \mathrm{m}(\text { mean } \pm S D)\end{array}$ & $80.80 \pm 2.38$ & $80.35 \pm 2.94$ & $81.49 \pm 3.59$ & -0.30 & 0.33 & 0.363 & -0.07 & 0.68 & 0.923 & 0.20 & 0.39 & 0.610 \\
\hline $\mathrm{RT}$ in & $324.47 \pm 13.24$ & $300.76 \pm 20.11$ & $306.6 \pm 17.99$ & -20.16 & 2.37 & $<0.001$ & -6.61 & 4.77 & 0.169 & -18.91 & 2.49 & $<0.001$ \\
\hline
\end{tabular}

AQP4-IgG, anti-aquaporin-4 antibody; B, estimate; GCIP, ganglion cell and inner plexiform layer; HC, healthy control; INL, inner nuclear layer; MOG-lgG, anti-myelin-oligodendrocyte-glycoprotein antibody; mRNFL, macular retinal nerve fibre layer; ONL, outer nuclear layer; OPL, outer plexiform layer; OPNL, outer plexiform and nuclear layer; PR, photoreceptor layer; $p R N F L$, peripapillary retinal nerve fibre layer; RT, total retinal thickness. 


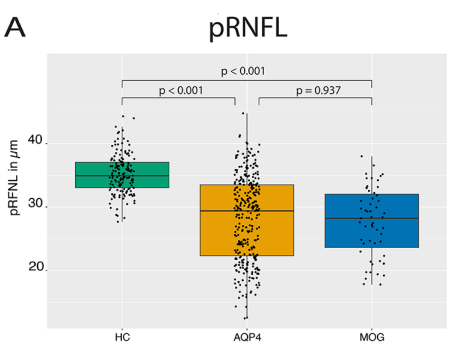

D

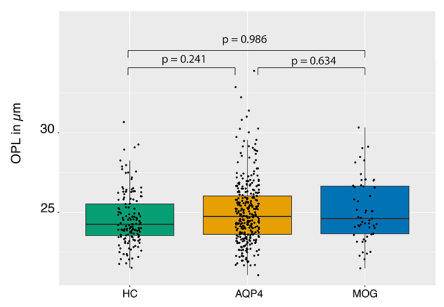

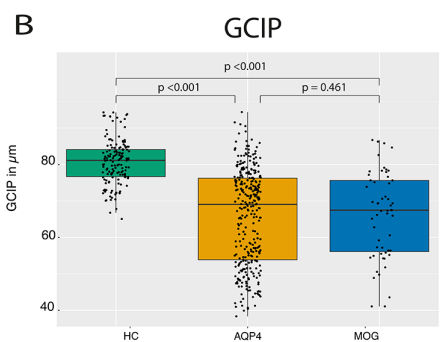

$\mathrm{E}$

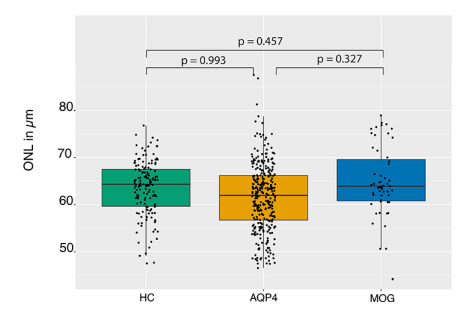

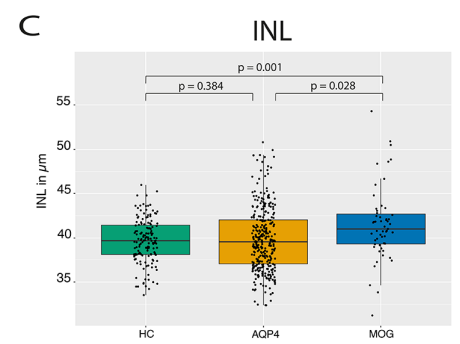

$\mathrm{F}$

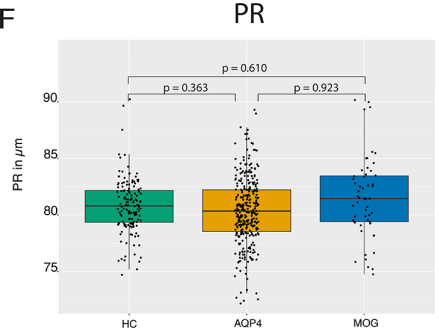

Figure 2 Group comparison of HC and patients who were AQP4-IgG and MOG-IgG seropositive at baseline: boxplots of mean OCT values with individual eyes (jitter) in HC (left, green), patients with AQP4-IgG (middle, yellow) and patients with MOG-IgG (right, blue). (A) pRNFL; (B) GCIP; (C) INL; (D) OPL; (E) ONL; and (F) PR. AQP4, aquaporin-4; HC, healthy control; GCIP, ganglion cell and inner plexiform layer; INL, inner nuclear layer; MOG, myelinoligodendrocyte-glycoprotein; OCT, optical coherence tomography; ONL, outer nuclear layer; OPL, outer plexiform layer; PR, photoreceptive layer; pRNFL, peripapillary retinal nerve fibre layer

models. Significance was established at $\mathrm{p}<0.05$. Statistical analyses were conducted using R (V.4.0.0) (RStudio Inc, Boston, Massachusetts, USA). ${ }^{27}$

\section{RESULTS}

\section{Cohort description}

In total, 197 patients who were AQP4-IgG seropositive fulfilled the inclusion criteria (figure 1, table 1). We also included 75 unmatched HCs and 32 patients who were MOG-IgG seropositiveas control groups.

Neuroaxonal damage measured by pRNFL, mRFNL and GCIP was comparable in patients who were AQP4-IgG seropositive (pRNFL: $78.46 \pm 24.13 \mu \mathrm{m}$, mRNFL: $28.09 \pm 6.60$ $\mu \mathrm{m}$, GCIP: $65.81 \pm 13.03 \mu \mathrm{m})$ and MOG-IgG seropositive (pRNFL: $74.33 \pm 23.44 \mu \mathrm{m}$, mRNFL: $27.62 \pm 5.43 \mu \mathrm{m}$, GCIP: $66.16 \pm 11.85 \mu \mathrm{m}$ ) making MOGAD a highly relevant comparative disease control group for our investigation of ORLs (table 2).

\section{Limited outer retinal changes in AQP4-IgG seropositive NMOSD}

No significant thinning of macular OPL and ONL in patients who were AQP4-IgG seropositive (irrespective of ON status) were observed compared with HC or patients who were MOGIgG seropositive using the $5 \mathrm{~mm}$ diameter macular data (table 2, figure 2). No significant changes were observed when the OPL and ONL values were analysed as the combined OPNL. Previous studies described ORL thinning only in the foveal and parafoveal area as a sign of AQP4-IgG-induced Müller cell damage. ${ }^{811}$ We therefore repeated our analyses in both $3 \mathrm{~mm}$ and the $1 \mathrm{~mm}$ diameter volumes around the fovea, but these narrower volumes showed again no relevant OPL or ONL thinning in patients who were AQP4-IgG seropositive compared with $\mathrm{HC}$ or patients who were MOG-IgG seropositive (see online supplemental data). Additionally, while these previous studies reported changes in the inner segment layer of the photoreceptors, this was not seen in our study. ${ }^{811}$

After a previous description ${ }^{11}$ of ORL changes in patients who were AQP4-IgG seropositive with a history of ON, we also examined ORL differences separately in eyes with a history of ON. AQP4-IgG seropositive eyes with a history of ON (AQP4ON) did not display any thinning of ONL and OPL compared with patients without a history of ON (AQP4-NON) or HC, despite severe neuroaxonal loss measured by pRNFL and GCIP layer (table 3, figure 3). Comparing patients who were AQP4-IgG and MOG-IgG seropositive, both groups had a comparable neuroaxonal loss (pRNFL, GCIP) - in the whole group as well as in respect of $\mathrm{ON}$ and non-ON eyes (table 2, figure 2). $\mathrm{AQP} 4-\mathrm{ON}(\mathrm{B}=-1.54, \mathrm{SE}=0.69 \mu \mathrm{m}, \mathrm{p}=0.027)$ as well as MOG-ON ( $\mathrm{B}=-2.51, \mathrm{SE}=0.87 \mu \mathrm{m}, \mathrm{p}=0.004)$ showed an OPL thinning in the fovea (1 $\mathrm{mm}$ diameter) compared with $\mathrm{HC}$, but no difference was observed between AQP4-ON and MOG-ON ( $p=0.100)$. Also, no significant correlation between ethnicity and current therapies on outer retinal thickness was found (data not shown).

\section{DISCUSSION}

Our study suggests that neither macular OPL nor ONL loss occurs in AQP4-IgG seropositive NMOSD, regardless of ON phenotype, as compared with $\mathrm{HC}$ and patients who were MOGIgG seropositive. The MOG-IgG cohort presented a unique opportunity to contrast our AQP4-IgG seropositive cohort with a highly relevant comparator group, which most likely has no astrocytopathy-component. ${ }^{28}$

Our results differ from those published by You et al in $2019^{8}$ and Filippatou et al in $2020 .{ }^{11}$ In both studies, thinning was observed in the ONL and the inner segment of the photoreceptor layers. In the case of You et al, who utilised Spectralis SD-OCT devices for the image acquisition, foveal thinning was observed along with a reduction in b-wave amplitudes in full-field electroretinography (ERG) suggestive of Müller cell dysfunction. ${ }^{8}$ Filippatou et al, who employed Cirrus-SD-OCT for the image acquisition, also described thinning of the fovea in the $5 \mathrm{~mm}$ diameter macular area around the fovea. ${ }^{11}$ Both studies suggested the ORL changes to be caused by a primary retinal astrocytopathy with AQP4-IgG associated glial dysfunction in Müller cells. ${ }^{29}$ These pathological responses could account for the associated thinning observed 
in the ONL in these studies. However, other exogenous factors cannot be ruled out as contributory, such as cohort composition and study methodologies.

On a cohort level, our population is larger (197 patients who were AQP4-IgG seropositive vs 22 and 51 by You et al and Filippatou et al, respectively) ${ }^{811}$ and more diverse than prior studies, which minimises potential type I errors. While You et al did not specify the ethnic composition of their cohort, the cohort in Filippatou et al had a relatively even distribution between Caucasian Americans (43\%) and African Americans (53\%) with a minor subset of Asian Americans (4\%)-describing a pronounced ONL thinning in African Americans. African American patients with multiple sclerosis (MS) are also known to suffer from faster and often more aggressive disease course in general, which could also be true for other neuroinflammatory diseases like NMOSD. ${ }^{30} 31$ Our AQP4-IgG seropositive cohort included an ethnically diverse dataset acquired worldwide with a lower African American patient composition (5.6\%), which might have contributed to the less profound foveal ONL changes. ${ }^{11} 32$

Recently, it has been hypothesised that the neuroplastic characteristics of the INL may act as a barrier to retrograde (but not anterograde) trans-synaptic axonal degeneration-rectified to the ORLs-in patients with MS following ON. ${ }^{33}$ This limited neuroplastic ability is hypothesised to rest with the bipolar, amacrine and horizontal cells, which feed into the synaptic tree at the level of the INL, and raises questions as to whether such protective mechanisms may also play a limited part in NMOSD and whether it remains so as we age. ${ }^{33}$ The average age of participants in the two other studies were relatively older (mean age for both being 47 years), whereas for our AQP4-IgG cohort it was 42 years. Previously reported studies concerning cohorts of similar demographic distribution to ours reported no significant correlation between age and retinal thickness. ${ }^{3435}$ However, agerelated changes in the retina cannot be ruled out and ORLs may be more susceptible to change with increasing age and/or disease duration. It is well-known that the plasticity of the CNS markedly reduces over time, and as a corollary, the regenerative properties of the INL may also be affected thereby diminishing its protective effects in reducing retrograde (trans-synaptic) axonal degeneration. ${ }^{36}$ The retina is also a vascularised organ, particularly at the interface between inner and outer retina, where the deep vascular plexus intercepts the boundary between the INL and OPL. ${ }^{37}$ Should the blood-retina barrier be compromised in the boundary between the INL and OPL, it is conceivable that the protective abilities of the INL may be circumvented and thereby mediating glial dysfunction in the Müller cells. This may have been what was observed in the OPL from the 1 $\mathrm{mm}$ AQP4-ON and MOG-ON cohort given the relative location of the OPL to the INL. To that end, while disease duration did not reveal to any correlates with OPL $(\mathrm{p}=0.805)$ or ONL $(p=0.835)$ values, we cannot exclude time-dependent effects in a cross-sectional analysis. We believe that this area warrants more research to quantify if (1) age is a factor, (2) ON damages the barrier function and (3) the INL does indeed play a role as a dam to retrograde axonal degeneration in NMOSD.

A strength of our study rests on its cohort size and composition, which mirrors that of a global population. This result derives from a consortium of expert NMOSD researchers enabling the enrolment of participants through a multicentre strategy. This approach was designed to overcome many of the earlier NMOSD study limitations, for example small and homogeneous sample populations. Additionally, the use of differing OCT devices compounds complexities in OCT comparisons and a high degree of caution is needed in order to rely on differing 
A

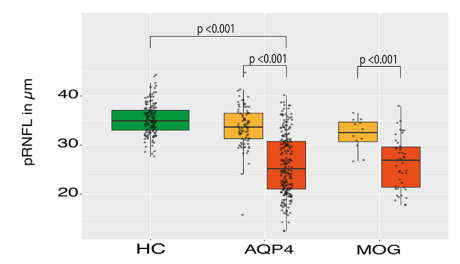

D

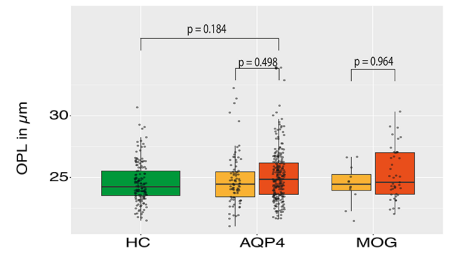

B

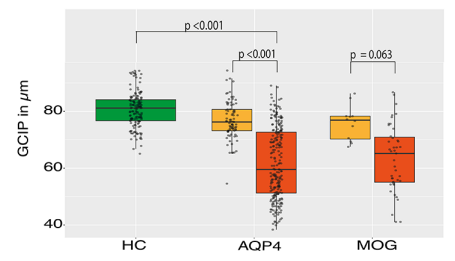

E

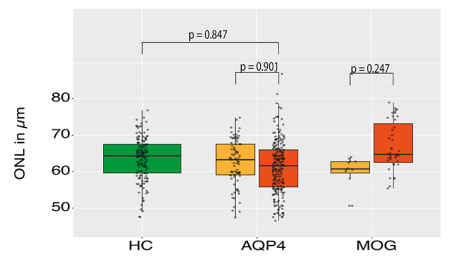

C

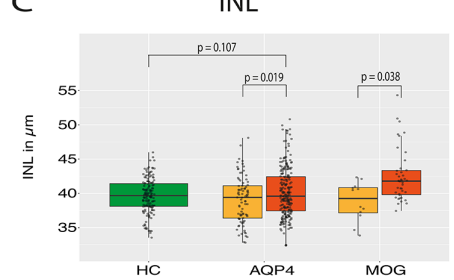

$\mathrm{F}$

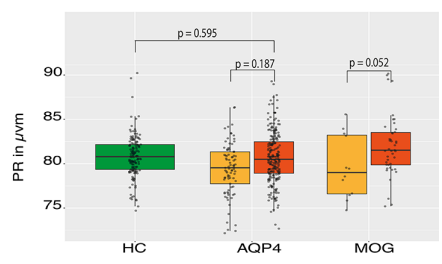

platforms interchangeably. ${ }^{38}$ Thus, our study focuses on use of three widely available OCT devices, and obtained confirmatory results with each of them; of these, two were also employed respectively in the studies by You et $a l^{8}$ and Filippatou et al. ${ }^{11}$

Limitations of the current study should also be considered. First, the HCs and patients with MOGAD were not matched, which makes it difficult to rule out age-related and genderrelated affects. Notably, retinal thickness decreases with age and males generally exhibit higher GCIP and RT. ${ }^{25}$ Also, no ERG or functional visual pathway assessments were conducted, which could have potentially shown more subtle functional impairment of ORLs without associated tissue loss. Outer retinal studies are additionally complicated by Henle Fibre morphologies as OCT beam placement plays a major role in how this layer is depicted; the high level of irregularity and variability in these morphologies add a level of subjectiveness in the quantification and correction of outer layer segmentation and analyses. ${ }^{39}$ Finally, Cirrus and Topcon measurements could not be utilised as confirmatory cohorts as there lacked sufficient HCs examined with these devices. Nonetheless, the current findings provide insights into relationships between retinal layer changes and axonal damage that have not previously been recognised; as no ORL changes can be observed on account of a primary astrocytopathy in NMOSD, it potentially alleviates the burden of monitoring the ORLs when tracking disease progression and reinforces the need to focus primarily on the inner layers, particularly the RNFL and the GCIP layer.

\section{CONCLUSION}

Our results show no evidence of macular ORL changes as a major component of retinal damage in patients who were seropositive AQP4-IgG NMOSD and patients with MOGAD. Further studies will be necessary to clarify (1) if OPL and ONL are damaged in late disease stages due to retrograde trans-synaptic axonal degeneration across the damaged INL barrier and (2) if outer retinal dysfunction without a measurable structural correlate occurs. Longitudinal studies could help quantify changes in the ORLs alongside disease progression.

\section{Author affiliations}

${ }^{1}$ Experimental and Clinical Research Center, Max Delbrück Center for Molecular Medicine and Charité - Universitätsmedizin Berlin, corporate member of Freie Universität Berlin and Humboldt-Universität zu Berlin, Berlin, Germany

${ }^{2}$ NeuroCure Clinical Research Center, Charité - Universitätsmedizin Berlin, corporate member of Freie Universität Berlin and Humboldt-Universität zu Berlin, Berlin, Germany

${ }^{3}$ CIEM MS Research Center, University of Minas Gerais State, Medical School, Belo Horizonte, Brazil

${ }^{4}$ Kashani MS Center, Isfahan University of Medical Sciences, Isfahan, Iran (the Islamic Republic of)

${ }^{5}$ School of Advanced Technologies in Medicine, Medical Image and Signal Processing Research Center, Isfahan University of Medical Sciences, Isfahan, Iran (the Islamic Republic of)

${ }^{6}$ Isfahan Eye Research Center, Department of Ophthalmology, Isfahan University of Medical Sciences, Isfahan, Iran (the Islamic Republic of)

${ }^{7}$ Center for Advanced Neurological Research, Nitte University, Mangalore, Karnataka, India

${ }^{8}$ Department of Neurology, National Cancer Center Korea, Goyang-si, Korea (the Republic of)

${ }^{9}$ Department of Opthalmology, Research Institute and Hospital of National Cancer Center, Goyang, Korea (the Republic of)

${ }^{10}$ Experimental Neurophysiology Unit, Institute of Experimental Neurology (INSPE) Scientific Institute, Hospital San Raffaele and University Vita-Salute San Raffaele,

Milano, Italy

${ }^{11}$ Division of Neurology, Department of Medicine, Siriraj Hospital and Bumrungrad International Hospital, Bangkok, Thailand

${ }^{12}$ Swedish Neuroscience Institute Neuro-Ophthalmology, Seattle, Washington, USA

${ }^{13}$ Department of Neurology, Neurology Service, University Hospital of Strasbourg, Strasbourg, France

${ }^{14}$ Department of Neurology, Oxford University Hospitals NHS Trust, Oxford, UK

${ }^{15}$ Department of Ophthalmology, Oxford University Hospitals NHS Trust, Oxford, UK

${ }^{16}$ Neuro-Opthalmology Division, Department of Opthalmology, Rabin Medical Center, Petah Tikva, Israel

${ }^{17}$ Sackler School of Medicine, Tel Aviv University, Tel Aviv, Israel

${ }^{18}$ Department of Neurology Slagelse, Institutes of Regional Health Research andMolecular Medicine, University of Southern Denmark, Odense, Syddanmark, Denmark

${ }^{19}$ Institute of Regional Health Research, University of Southern Denmark, Odense, Denmark

${ }^{20}$ Hospital Clinic of Barcelona-Institut d'Investigacions, Biomèdiques August Pi Sunyer, University of Barcelona, Barcelona, Spain

${ }^{21}$ Institute of Clinical Neuroimmunology, LMU Hospital, Ludwig-MaximiliansUniversitat Munchen, Munich, Germany

${ }^{22}$ Department of Neurology, University of Michigan Medical School, Ann Arbor, Michigan, USA 
${ }^{23}$ NYU Multiple Sclerosis Comprehensive Care Center, Department of Neurology, NYU, New York, New York, USA

${ }^{24}$ Neurology, Multiple Sclerosis, Myelin Disorders and Neuroinflammation, Hospital for Neurology Pierre Wertheimer, Lyon, France

${ }^{25}$ Centre d'Esclerosi Múltiple de Catalunya (Cemcat). Department of Neurology/ Neuroimmunology, Universitat Autònoma de Barcelona, Barcelona, Spain

${ }^{26}$ Department of Neurology, Koc University Research Center for Translational Medicine (KUTTAM), Koc University School of Medicine, Istanbul, Turkey

${ }^{27}$ Cerrahpaşa Faculty of Medicine, Department of Neurology, Istanbul UniversityCerrahpaşa, Istanbul, Turkey

${ }^{28}$ Department of Ophthalmology, Cerrahpasa Medical Faculty, Istanbul Universitesi, Fatih, Turkey

${ }^{29}$ Department of Neurology, Medical Faculty, Heinrich-Heine-Universitat Dusseldorf, Dusseldorf, Nordrhein-Westfalen, Germany

Department of Neurology, Center for Neurology and Neuropsychiatry, LVRKlinikum, Heinrich-Heine-Universitat Dusseldorf, Dusseldorf, Germany

${ }^{31}$ Department of Ophthalmology and Visual Sciences, Escola Paulista de Medicina, Universidade Federal de São Paulo, São Paulo, Brazil

${ }^{32}$ Department of Neurology and Neurosurgery, Escola Paulista de Medicina,

Universidade Federal de São Paulo, Sao Paulo, Brazil

${ }^{33}$ The Walton Centre NHS Foundation Trust, Liverpool, UK

${ }^{34}$ Department of Neurology, Hospital Clinico de Maracaibo, Maracaibo, Venezuela, Bolivarian Republic of

${ }^{35}$ Moorfield's Eye Hospital, The National Hospital for Neurology and Neurosurgery, Queen Square Institute of Neurology, University College London, London, UK

${ }^{36}$ Department of Neurology, University of California San Francisco, San Francisco, California, USA

${ }^{37}$ Department of Medicine, Harbor-University of California at Los Angeles (UCLA) Medical Center, and Lundquist Institute for Biomedical Innovation, Torrance, California, USA

${ }^{38}$ Department of Medicine, David Geffen School of Medicine, UCLA, Los Angeles, California, USA

${ }^{39}$ Departments of Ophthalmology and Visual Sciences, Kellogg Eye Center, Ann Arbor, Michigan, USA

${ }^{40}$ Department of Metabolism, Endocrine and Diabetes, Department of Interna Medicine, University of Michigan Medical School, Ann Arbor, Michigan, USA

${ }^{41}$ Department of Pediatrics, University of Utah Health, Salt Lake City, Utah, USA

${ }^{42}$ Department of Neurology, Charité - Universitätsmedizin Berlin, corporate member

of Freie Universität Berlin and Humboldt-Universität zu Berlin, Berlin, Germany

${ }^{43}$ Department of Neurology, University of California Irvine, Irvine, California, USA

Twitter Elena H Martinez-Lapiscina @elenahlapiscina@ and Joachim Havla @ NeuroVisionLab

Contributors The study was conceived and designed by $\mathrm{AL}, \mathrm{HZ}, \mathrm{FCO}, \mathrm{AB}$ and $\mathrm{FP}$ $A L, H Z, F C O, C B, S M$ and SuS collected and analysed data for the study. $H Z$, FCO, $A B$ and $F P$ contributed to the scientific design and conduct of the study. $A L$ and FCO performed biostatistical analyses to the study, were responsible for literature research and wrote the manuscript. AP, AJG, MRY, LC and TJS contributed to the conception and design of the study. All other authors contributed to the acquisition and analysis of data. All authors approved the final version of the manuscript.

Funding The authors acknowledge support from the Guthy Jackson Charitable Foundation (GJCF) and the German Research Foundation (DFG) pertaining to the CROCTINO project.

Competing interests $\mathrm{HZ}$ reports grants from Novartis and speaking honoraria from Bayer Healthcare, unrelated to this study. EHM-L received funding from the Instituto de Salud Carlos III (Spain) and Fondo Europeo de Desarrollo Regional (FEDER-JR16/00006), Grant for MS Innovation, Fundació Privada Cellex and Marató TV3 Charitable Foundation and is a researcher in the OCTIMS study, an observational study (that involves no specific drugs) to validate SD-OCT as a biomarker for MS, sponsored by Novartis and has received honoraria and travel support for international and national meetings over the last 3 years from from Biogen, Novartis, Roche, Genzyme. She is a member of the working committee of International Multiple Sclerosis Visual System (IMSVISUAL) Consortium. MAL-P has received funding for travel and speaker honoraria from Novartis, SanofiGenzyme and Roche. MAF has nothing to disclose. Jacqueline Palace has received support for scientific meetings and honorariums for advisory work From Merck Serono, Novartis, Chugai, Alexion, Roche, Medimmune, Argenx, UCB, Mitsubishi, Amplo, Janssen. Grants from Alexion, Amplo biotechnology. Shares in AstraZenica. Acknowledges Partial funding by Highly specialised services NHS England. MIL reported being involved in aquaporin 4 testing, receiving salary from the National Health Service National Highly Specialised Commissioning Group for Neuromyelitis Optica, UK, being supported by the National Institute for Health Research Oxford Biomedical Research Centre, UK, and receiving speaking honoraria and travel grants from Biogen Idec, and travel grant from Novartis. SMS has nothing to disclose. AR-F is sponsored by Abide Therapeutic outside of the submitted work and reports no potential conflicts of interest. SSiritho received funding for travel and speaker honoraria from Merck Serono, Pacific Healthcare (Thailand), Menarini (Thailand), Biogen Idec, UCB (Thailand), and Novartis. AA reports personal fees from received honoraria for giving educational presentations on multiple sclerosis and neuroimmunology at several national congresses or symposia from Teva Turkey, Merck-Serono, Biogen Idec-Gen Pharma of Turkey, Roche, Novartis, Bayer, Sanofi-Genzyme. She has received travel and registration coverage for attending several national and international congresses or symposia from Merck-Serono, Biogen Idec-Gen Pharma of Turkey, Roche, Sanofi-Genzyme and Bayer. AJ has received compensation for advisory board, consulting, meeting attendance and speaking from Biogen, Terumo-BCT, Genentech, Shire and Chugai Pharmaceuticals. $\mathrm{SH}$ has received funding from the NMO Spectrum-UK charity and was previously funded by an MGA/Watney/NIHR Oxford Biomedical research grant. RM serves on scientific advisory board for MedImmune and has received funding for travel and honoraria from Biogen, Merck Serono, Novartis, Sanofi- Genzyme, Roche and Teva. EN has nothing to disclose. ACC received funding from the Instituto de Salud Carlos III (Spain) JR19/00007 unrelated to this manuscript. DB has received speaking/ consulting honoraria from Bayer Health Care, Biogen Idec, Merck, Sanofi-Genzyme, TEVA and Roche and had travel expenses to scientific meetings sponsored by Bayer Health Care, Merck Serono, TEVA and Roche. JH reports grants for OCT research from the Friedrich-Baur-Stiftung and Merck, personal fees and non-financial support from Celgene, Merck, Alexion, Novartis, Roche, Santhera, Biogen, Heidelberg Engineering, Sanofi Genzyme and non-financial support of the Guthy-Jackson Charitable Foundation, all outside the submitted work. JH is partially funded by the German Federal Ministry of Education and Research (DIFUTURE), Grant Numbers 01ZZ1603[A-D] and 01ZZ1804[A-H]. LL received honoraria for consulting services from Merck, Roche, Biogen and for speaking activities from Teva; research support from Merck, Biogen, Novartis; travel support from Merck, Roche, Biogen, Almirall. MP has nothing to disclose. OA has received honoraria for speaking/consultation and travel grants from Bayer Healthcare, Biogen Idec, Chugai, Novartis, Medimmune, Merck Serono, and Teva and research grants from Bayer Healthcare, Biogen Idec, Novartis, and Teva. MR received speaker honoraria from Novartis, Bayer, Roche, Alexion and Ipsen and travel reimbursement from Bayer, Biogen, Merz, Genzyme, Teva, Roche and Merck, none related to this study. PA reports grants, personal fees and non-financial support from Allergan, Biogen, Ipsen, Merz Pharmaceuticals, Novartis, and Roche, personal fees and non-financial support from Bayer Healthcare, and Merck, and non-financial support from Sanofi-Aventis/Genzyme. HJK reports speaking and/or consulting: Bayer Schering Pharma, Biogen, Celltrion, Eisai, HanAll BioPharma, Medlmmune, Merck Serono, Novartis, Sanofi Genzyme, Teva-Handok, and UCB; research support: Ministry of Science \& ICT, Sanofi Genzyme, Teva-Handok, and UCB; steering committee member: MedImmune; co-editor/associated editor: MS Journal-Experimental, Translational and Clinical; and Journal of Clinical Neurology. J-WH has received a grant from the National Research Foundation of Korea. YM-D has served as a consultant and/or received grant support from: Acorda, Bayer Pharmaceutical, Biogen Idec, Celgene, EMD Serono, Genzyme, Novartis, Questor, Chugai, and Teva Neuroscience and is currently supported by grants from NIH NIAID Autoimmune Center of Excellence: UM1-Al110557; NIH NINDS R01-NS080821. HSK has nothing to disclose. IK served on scientific advisory board for Biogen Idec and Genentech and received research support from Guthy-Jackson Charitable Foundation, National Multiple Sclerosis Society, Biogen-Idec,Serono, Genzyme and Novartis. ZR has nothing to disclose. AR has nothing to disclose. MRY is founder and a shareholder of NovaDigm Therapeutics, Inc; he receives funding from the United States National Institutes of Health and United States Department of Defense; he holds US and international patents on immunotherapeutic and anti-infective technologies, is a member of the Genentech-Roche Scientific Advisory Committee and adviser to The Guthy-Jackson Charitable Foundation. TJS was issued US patents covering the therapeutic targeting of IGF-I receptor in autoimmune diseases. He is a paid consultant for Horizon Thera and Immunovant and is a scientific advisor to the Guthy-Jackson Charitable Foundation. He receives research funding from the National Institutes of Health. AP is supported by the National Institute for Health Research (NIHR) Biomedical Research Centre based at Moorfields Eye Hospital National Health Service (NHS) Foundation Trust and University College London Institute of Ophthalmology. AB is cofounder and shareholder of Motognosis and Nocturne. He is named as inventor on several patent applications regarding MS serum biomarkers, OCT image analysis and perceptive visual computing. FP reports research grants and speaker honoraria from Bayer, Teva, Genzyme, Merck, Novartis, Medlmmune and is member of the steering committee of the OCTIMS study (Novartis), all unrelated to this work. FCO was employee of Nocturne GmbH and receives research support by the American Academy of Neurology and National Multiple Sclerosis Society (US), unrelated to this work as well as funding by the German Association of Neurology (Deutsche Gesellschaft für Neurologie) in context of this project.CC has received a speaking honorarium from Bayer and research funding from Novartis unrelated to this publication. All other authors have nothing to disclose.

Patient consent for publication Consent obtained directly from patient(s)

Ethics approval Written informed consent was obtained from all patients prior to the commencement of the study and institutional review board approvals for retrospective data use were obtained or waived from each centre in accordance with 
the Declaration of Helsinki (1964) in its currently applicable version at their own discretion and in accordance with relevant local laws. The study also conformed to all relevant best practice guidelines and ethical standards of each centre.

Provenance and peer review Not commissioned; externally peer reviewed.

Data availability statement Data are available upon reasonable request. All authors commit to sharing anonymised data pertaining to this study to the BMJ as reasonably requested and subject to the ICMJE data sharing policy of 1 July 2018.

Supplemental material This content has been supplied by the author(s). It has not been vetted by BMJ Publishing Group Limited (BMJ) and may not have been peer-reviewed. Any opinions or recommendations discussed are solely those of the author(s) and are not endorsed by BMJ. BMJ disclaims all liability and responsibility arising from any reliance placed on the content. Where the content includes any translated material, BMJ does not warrant the accuracy and reliability of the translations (including but not limited to local regulations, clinical guidelines, terminology, drug names and drug dosages), and is not responsible for any error and/or omissions arising from translation and adaptation or otherwise.

Open access This is an open access article distributed in accordance with the Creative Commons Attribution Non Commercial (CC BY-NC 4.0) license, which permits others to distribute, remix, adapt, build upon this work non-commercially, and license their derivative works on different terms, provided the original work is properly cited, appropriate credit is given, any changes made indicated, and the use is non-commercial. See: http://creativecommons.org/licenses/by-nc/4.0/.

\section{ORCID iDs}

Angelo Lu http://orcid.org/0000-0002-7897-6498

Claudia Chien http://orcid.org/0000-0001-8280-9513

Ho Jin Kim http://orcid.org/0000-0002-8672-8419

Adriana Roca-Fernández http://orcid.org/0000-0002-8720-9397

Hadas Stiebel-Kalish http://orcid.org/0000-0001-7715-6706

Elena H Martinez-Lapiscina http://orcid.org/0000-0003-4272-0826

Joachim Havla http://orcid.org/0000-0002-4386-1340

Marius Ringelstein http://orcid.org/0000-0003-3618-8407

Philipp Albrecht http://orcid.org/0000-0001-7987-658X

Denis Bernardi Bichuetti http://orcid.org/0000-0002-4011-3734

Axel Petzold http://orcid.org/0000-0002-0344-9749

Frederike Cosima Oertel http://orcid.org/0000-0003-4906-5983

\section{REFERENCES}

1 Jarius S, Paul F, Weinshenker BG, et al. Neuromyelitis optica. Nat Rev Dis Primers 2020;6:85.

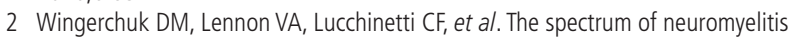
optica. Lancet Neurol 2007:6:805-15.

3 Oertel FC, Kuchling J, Zimmermann $\mathrm{H}$, et al. Microstructural visual system changes in AQP4-antibody-seropositive NMOSD. Neurol Neuroimmunol Neuroinflamm 2017;4:e334.

4 Uzawa A, Mori M, Kuwabara S. Neuromyelitis optica: concept, immunology and treatment. J Clin Neurosci 2014;21:12-21.

5 Papadopoulos MC, Verkman AS. Aquaporin 4 and neuromyelitis optica. Lancet Neurol 2012;11:535-44.

6 Nagelhus EA, Ottersen OP. Physiological roles of aquaporin-4 in brain. Physiol Rev 2013;93:1543-62.

7 Bringmann A, Pannicke T, Grosche J, et al. Müller cells in the healthy and diseased retina. Prog Retin Eye Res 2006;25:397-424.

8 You Y, Zhu L, Zhang T, et al. Evidence of Müller glial dysfunction in patients with aquaporin-4 immunoglobulin G-Positive neuromyelitis optica spectrum disorder. Ophthalmology 2019;126:801-10.

9 Jeong $\mathrm{IH}$, Kim HJ, Kim N-H, et al. Subclinical primary retinal pathology in neuromyelitis optica spectrum disorder. J Neurol 2016;263:1343-8.

10 Oertel FC, Outteryck O, Knier B, et al. Optical coherence tomography in myelinoligodendrocyte-glycoprotein antibody-seropositive patients: a longitudinal study. $J$ Neuroinflammation 2019:16:154.

11 Filippatou AG, Vasileiou ES, He Y, et al. Evidence of subclinical quantitative retinal layer abnormalities in AQP4-IgG seropositive NMOSD. Mult Scler 2021;27:1738-48.

12 Oertel FC, Havla J, Roca-Fernández A, et al. Retinal ganglion cell loss in neuromyelitis optica: a longitudinal study. J Neurol Neurosurg Psychiatry 2018;89:1259-65.

13 Motamedi S, Oertel FC, Yadav SK, et al. Altered fovea in AQP4-IgG-seropositive neuromyelitis optica spectrum disorders. Neurol Neuroimmunol Neuroinflamm 2020;7. doi:10.1212/NXI.0000000000000805. [Epub ahead of print: 23 Jun 2020].
14 Specovius S, Zimmermann HG, Oertel FC, et al. Cohort profile: a collaborative multicentre study of retinal optical coherence tomography in 539 patients with neuromyelitis optica spectrum disorders (CROCTINO). BMJ Open 2020;10:e035397.

15 Oertel FC, Specovius S, Zimmermann HG, et al. Retinal optical coherence tomography in neuromyelitis optica. Neurol Neuroimmunol Neuroinflamm 2021;8. doi:10.1212/ NXI.0000000000001068. [Epub ahead of print: 15 Sep 2021].

16 Bruijstens AL, Wong YYM, van Pelt DE, et al. Hla association in MOG-IgG- and AQP4IgG-related disorders of the CNS in the Dutch population. Neurol Neuroimmunol Neuroinflamm 2020;7. doi:10.1212/NXI.0000000000000702. [Epub ahead of print: 20 Mar 2020].

17 Kim H, Lee E-J, Kim S, et al. Serum biomarkers in myelin oligodendrocyte glycoprotein antibody-associated disease. Neurol Neuroimmunol Neuroinflamm 2020;7. doi:10.1212/NXI.0000000000000708. [Epub ahead of print: 17 Mar 2020].

18 Narayan R, Simpson A, Fritsche K, et al. Mog antibody disease: a review of MOG antibody seropositive neuromyelitis optica spectrum disorder. Mult Scler Relat Disord 2018;25:66-72.

19 Havla J, Kümpfel T, Schinner R, et al. Myelin-oligodendrocyte-glycoprotein (MOG) autoantibodies as potential markers of severe optic neuritis and subclinical retinal axonal degeneration. J Neurol 2017;264:139-51.

20 Reindl $M$, Schanda $K$, Woodhall $M$, et al. International multicenter examination of MOG antibody assays. Neurol Neuroimmunol Neuroinflamm 2020;7:e674.

21 Waters P, Reindl M, Saiz A, et al. Multicentre comparison of a diagnostic assay: aquaporin-4 antibodies in neuromyelitis optica. J Neurol Neurosurg Psychiatry 2016:87:1005-15

22 Tewarie P, Balk L, Costello F, et al. The OSCAR-IB consensus criteria for retinal OCT quality assessment. PLoS One 2012;7:e34823.

23 Schippling S, Balk LJ, Costello F, et al. Quality control for retinal OCT in multiple sclerosis: validation of the OSCAR-IB criteria. Mult Scler 2015;21:163-70.

24 Aytulun A, Cruz-Herranz A, Aktas 0, et al. Apostel 2.0 recommendations for reporting quantitative optical coherence tomography studies. Neurology 2021;97:68-79.

25 Motamedi S, Gawlik K, Ayadi N, et al. Normative data and minimally detectable change for inner retinal layer thicknesses using a semi-automated OCT image segmentation pipeline. Front Neurol 2019;10:1117

26 Staurenghi G, Sadda S, Chakravarthy U, et al. Proposed lexicon for anatomic landmarks in normal posterior segment spectral-domain optical coherence tomography: the IN•OCT consensus. Ophthalmology 2014;121:1572-8.

27 R Core Team. R: a language and environment for statistical computing. Vienna, Austria: R Foundation for Statistical Computing, 2017. https://www.R-project.org/

28 Höftberger R, Guo Y, Flanagan EP, et al. The pathology of central nervous system inflammatory demyelinating disease accompanying myelin oligodendrocyte glycoprotein autoantibody. Acta Neuropathol 2020;139:875-92.

29 Goodyear MJ, Crewther SG, Junghans BM. A role for aquaporin-4 in fluid regulation in the inner retina. Vis Neurosci 2009;26:159-65.

30 Caldito NG, Saidha S, Sotirchos ES, et al. Brain and retinal atrophy in AfricanAmericans versus Caucasian-Americans with multiple sclerosis: a longitudinal study. Brain 2018;141:3115-29.

31 Mealy MA, Kessler RA, Rimler Z, et al. Mortality in neuromyelitis optica is strongly associated with African ancestry. Neurol Neuroimmunol Neuroinflamm 2018;5:e468.

32 Liu J, Mori M, Zimmermann H, et al. Anti-Mog antibody-associated disorders: differences in clinical profiles and prognosis in Japan and Germany. J Neurol Neurosurg Psychiatry 2021;92:377-83.

33 Panneman EL, Coric D, Tran LMD, et al. Progression of anterograde trans-synaptic degeneration in the human retina is modulated by axonal convergence and divergence. Neuroophthalmology 2019;43:382-90

34 Tian G, Li Z, Zhao G, et al. Evaluation of retinal nerve fiber layer and ganglion cell complex in patients with optic neuritis or neuromyelitis optica spectrum disorders using optical coherence tomography in a Chinese cohort. J Ophthalmol 2015;2015:1-6.

35 Martinez-Lapiscina EH, Sepulveda M, Torres-Torres R, et al. Usefulness of optical coherence tomography to distinguish optic neuritis associated with AQP4 or MOG in neuromyelitis optica spectrum disorders. Ther Adv Neurol Disord 2016;9:436-40.

36 Balk LJ, Coric D, Knier B, et al. Retinal inner nuclear layer volume reflects inflammatory disease activity in multiple sclerosis; a longitudinal OCT study. Mult Scler J Exp Trans/ Clin 2019:5:205521731987158.

37 Campbell JP, Zhang M, Hwang TS, et al. Detailed vascular anatomy of the human retina by Projection-Resolved optical coherence tomography angiography. Sci Rep 2017;7:42201

38 Bhargava $\mathrm{P}$, Lang A, Al-Louzi O, et al. Applying an open-source segmentation algorithm to different OCT devices in multiple sclerosis patients and healthy controls: implications for clinical trials. Mult Scler Int 2015;2015:1-10.

39 Gonzalez Caldito N, Antony B, He Y, et al. Analysis of agreement of Retinal-Layer thickness measures derived from the segmentation of horizontal and vertical spectralis OCT macular scans. Curr Eye Res 2018;43:415-23. 